\title{
Performance Tuning of Spade Card Antenna using Mean Average Loss of Backpropagation Neural Network
}

\author{
Irfan Mujahidin $^{1}$, Dwi Arman Prasetya ${ }^{2}$, Nachrowie ${ }^{3}$, Samuel Aji Sena ${ }^{4}$, Putri Surya Arinda ${ }^{5}$ \\ Department of Electrical Engineering, University of Merdeka Malang, \\ Malang, Indonesia ${ }^{1,2,3,4,5}$
}

\begin{abstract}
The microstrip antennas have different dimensions to get the desired performance, especially for microstrip antennas that have complex components and dimensions with the performance: the range of frequency at $2.4 \mathrm{GHz}$ until $3.6 \mathrm{GHz}$, Maximum Power of Gain value is $5.83 \mathrm{~dB}$ and the minimum value is $3 \mathrm{~dB}$ and Maximum Directivity Value is 6.22 and the minimum value is 3.32 . in consequence, needs to fill the demand for a new and the corresponding design as solvent to adaptive matching as tuner the frequency on antenna design that needs requires a complex mathematical method and simulation. This paper has the novel design to tune the performance of spade card microstrip antenna that can operate on the single, dual or multiband and able to produce circular or linear polarization using Backpropagation Neural Network in order to obtain an optimum design with a backpropagation algorithm as a solution to simplify the design process. As a result, after 20000 epochs the training loss is around 0.044 and the testing loss is around 0.058 . The model has a good performance despite only using a few numbers of training data.
\end{abstract}

Keywords-Spade card antenna; mean average loss; neural network; performance tuning antenna

\section{INTRODUCTION}

The progress of telecommunication technology has developed very rapidly. This can be seen from the increasing need to obtain information, whether the information in the form of sound, data, pictures, or video, with communication equipment that can be used anywhere and anytime. The most developed technological advancement today is wireless communication, which can support the implementation of a global telecommunication system. One example of the application of wireless communication is WLAN (Wireless Local Area Network) that can meet the needs of access to information and communication that can be used anywhere and anytime[1]. Compared to the Wired Local Area Network that used to use the cable as its transmission medium, WLAN technology is easier in terms of installation, practical and efficient[2].

In the application of wireless communication, the role of the antenna as an electromagnetic component is necessary because of the antenna attend as a means to emit and take electromagnetic waves in which the equipment formation signal is contained. The antenna is a very important component to support the wireless communication system[3]. The latest developments in wireless communication systems require antenna characteristics that have relatively small, flexible and practical shapes.

The microstrip antenna is one kind of the antenna with the exact quality of the required telecommunication circuit. The microstrip antenna is made on a specific substrate material with a radiating element located on one side of the substrate and the other is a conductor layer that acts as a ground plane[4]. Microstrip antennas work on UHF frequency allocations (300 $\mathrm{MHz}-3000 \mathrm{MHz}$ ) up to X Band (5200 MHz - $10900 \mathrm{MHz}$ ) so that microstrip antennas can be used for wireless communication.

Microstrip antennas have different components and dimensions to get different performance after what is needed, especially for microstrip antennas that have complex dimensions and components[5]. in consequence, needs to fill the demand for a new and the corresponding design as solvent to adaptive matching as tuner the frequency on antenna design that needs requires a complex mathematical method and simulation. This paper has the novel design to tune the performance of spade card microstrip antenna that can operate on the single, dual or multi-band and able to produce circular or linear polarization using Backpropagation Neural Network [6][7]. The model has a good performance despite only using a few numbers of training data.

\section{RESEARCH METHOD}

\section{A. Antenna Design}

The antenna constructed in this paper is a microstrip circuit antenna with the following component specifications:

- Dielectric material : FR4

The dielectric value $(\mathrm{gr}) \quad=4.2$

The dielectric components thickness $(\mathrm{h})=1.6 \mathrm{~mm}$

- Seam substrate copper :

The conductor material thickness $(\mathrm{t}) \quad=0.000001 \mathrm{~mm}$

Copper conductivity $(\sigma)$ $=5.80 \times 107 \mathrm{mho}$ $\mathrm{m}-1$

Material size

$=29.7 \times 21 \mathrm{~mm}$

After mathematically calculated this research did a simulation and optimization to get the expected results using 
CST software. The shape of the ground plane and patches are made to have different sizes to obtain the desired frequency[8]. Shape design and simulation of this antenna is important because it will affect the antenna performance.
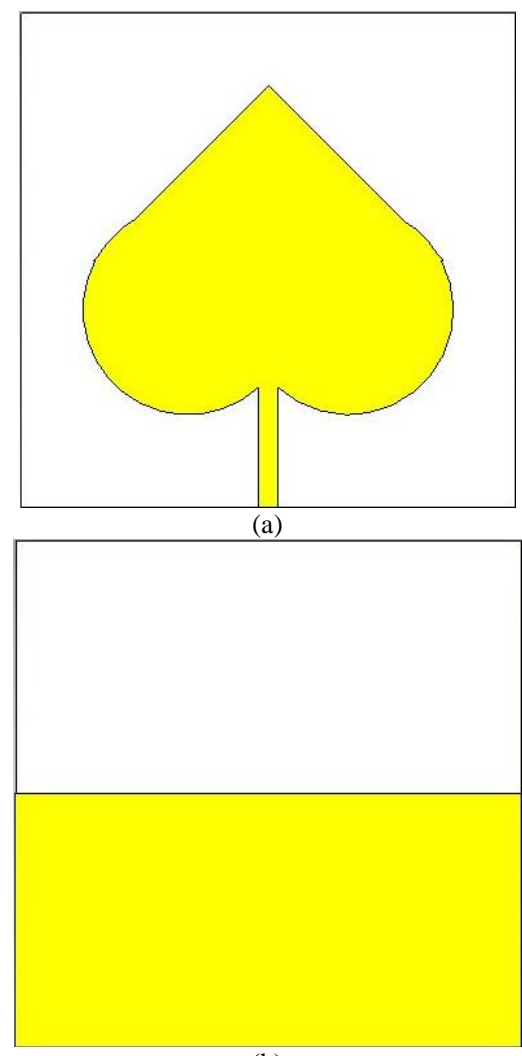

(b)

Fig. 1. Geometric Spade Card Shapes Patch Microstrip Antenna Front (b) Rear

The main part of the antenna is a triangle shape joined with a double circle at the bottom that resembles the shape of a reversed heart, shown in Fig. 1. Part circle on the patch directly connect the feeder line as a link to the patch on the antenna connector[9]. Then the ground plane consists from one form and forms ground plane is basically that is rectangular in addition to the connection with the connector.

In this paper, basically the patch of the antenna has the circular shape, so the component of patch element dimensions with the equation:

$$
\alpha=\frac{\mathrm{F}}{\left\{1+\frac{2 \mathrm{~h}}{\pi \varepsilon_{\mathrm{r}} \mathrm{F}}\left[\ln \left(\frac{\pi \mathrm{F}}{2 \mathrm{~h}}\right)+1.7726\right]\right\}^{\frac{1}{2}}}
$$

The notation above can be explained that a is the radius of the antenna patch component, $\mathrm{h}$ is the substrate thickness, $\mathrm{\varepsilon r}$ is the dielectric permittivity of the substrate and $F$ is the logarithmic function. The transmission line circuit of microstrip element design is finished theoretical based on the matter and any literature [10][11].

\section{B. Datasets}

The data used in this paper have been obtained from the CST design results. The antenna simulation using a different combination of ground plane height and the triangle and circle dimensions resulted in 123 samples which have been classified to be more precise. in this research, picked random 90 samples which have been measured and used it as the training data and the rest as the testing data[12][13]. The frequency, return loss, gain and directivity will be saved for further use.

\section{Network Achitecture}

The implementation uses a three-layer of a Multilayer Perceptron in this paper. In Fig. 2, The input layer has only one node as the frequency, 16 nodes inside the hidden layer to add a non-linearity to model and 6 nodes in the output layer as the representation of the ground plane height, triangle side length, circle diameters, return loss, gain and directivity of the antenna[14][15].

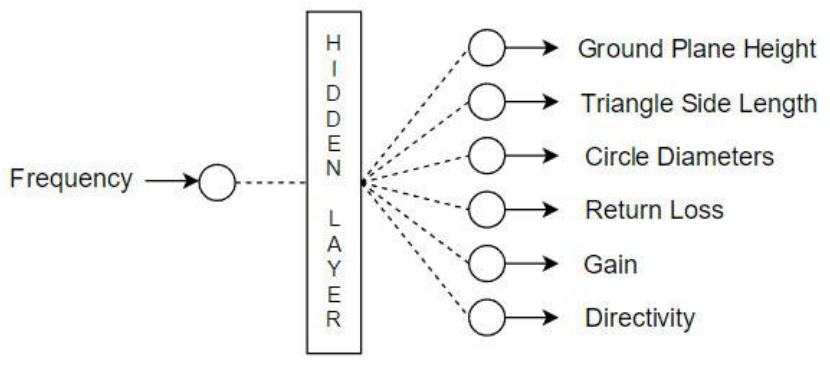

Fig. 2. Neural Network Architecture

The algorithm was experimented using a different-numbers of hidden layers and neurons[16][17]. However, the architecture explained above is the best possible combination and has the best result in minimizing the loss and converge faster than any other combination.

\section{Implementation}

The training process of backpropagation network consists of two main step. First, training features will be fed to the input layer and will flow through all neuron in the hidden layer. The interconnection between neuron is called synapse. All synapse in the network has its own weight[18][19]. The features will be multiplied with its synapse weight and add a bias value at the same time. After the features arrive at the output layer, the loss will be computed using Mean Average Loss (MAE) (1). Second, the loss value will be minimized using stochastic gradient descent. The loss value will be backpropagated to compute the gradient to minimize the loss and update all synapse weights.

$$
\text { MAE }=\frac{1}{n} \sum_{j=1}^{n}\left|y_{j}-\hat{y}_{j}\right|
$$

Neural network model was created using Python and Tensor flow framework. The activation function of all neuron inside the hidden layer are ReLU and the activation function of the output are Linear, because the method do regression task in this experiment[20][21]. Stochastic Gradient Descent (SGD) used as the optimizer with learning rate of 0.0001 and repeated until 20.000 epochs. Training data will feed into the network in batch of 10 data. Thus to run experiments, we compare the results of several experimental samples with the accuracy of the prediction algorithm used. Thus, to measure the success of 
the implementation of the method used by comparing the results of experiments and comprehensive algorithm predictions.

\section{RESULT AND ANALYSIS}

After 20.000 Epochs, the analysis evaluates the model using the test data. The analysis evaluates the MAE value and compare that value with the loss of the training process. The training loss is around 0.044 and the testing loss is around 0.058 .

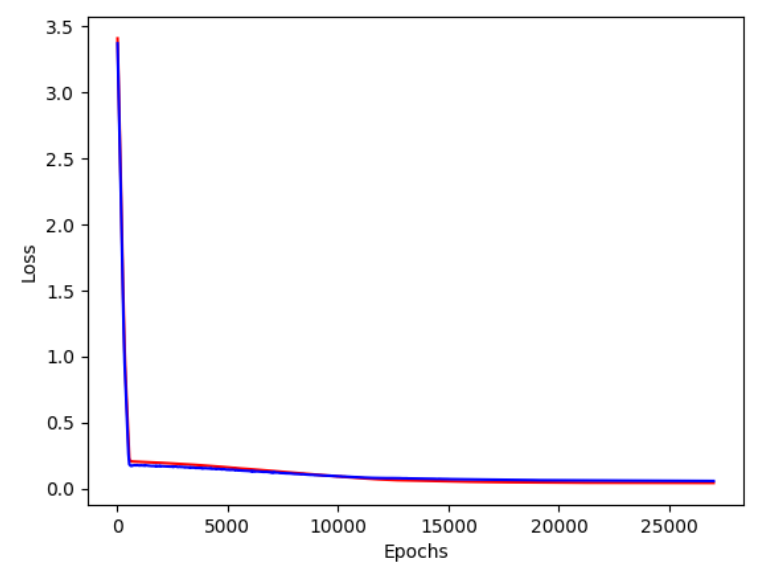

Fig. 3. Training and Testing Loss

Based on Fig. 3 the results provide the comparison of testing data with the output of the neural network for input frequency of $2913 \mathrm{MHz}$ in Table I. The model can predict the dimension of the antenna with a very low error [22][23]. But there are some gap in the S-Parameter, Gain and Directivity estimation. Thus, a better quantity and quality of a new dataset are required for further research. To know more results from the results of sampling data can be seen in Fig. 4 in the attachment.

TABLE I. COMPARISON OF PREDICTION AND TRUTH FOR 2913 MHZ INPUT

\begin{tabular}{|l|l|l|}
\hline Parameters & Truth & Prediction \\
\hline Ground Plane Height & 40.1 & 40.1 \\
\hline Triangle Side Length & 20 & 20 \\
\hline Circle Diameters & 17.300 & 17.333 \\
\hline S-Parameter & -20.230 & -21.076 \\
\hline Gain & 4.4 & 4.821 \\
\hline Directivity & 5.420 & 5.364 \\
\hline
\end{tabular}

\section{CONCLUSION}

Backpropagation neural network has been used to design an antenna. By using a correct activation function and proper learning rate, backpropagation neural network shows a promising result for the tune of a spade card shape microstrip antenna: the range of frequency at $2.4 \mathrm{GHz}$ until $3.6 \mathrm{GHz}$, Maximum Power of Gain value is $5.83 \mathrm{~dB}$ and the minimum value is $3 \mathrm{~dB}$ and Maximum Directivity Value is 6.22 and the minimum value is 3.32 . The model has a good performance despite only using a few numbers of training data. This research can be pushed further to learn using a different shape, substrate, frequency range and other parameters of a microstrip antenna. For future work it is necessary to develop a wider range of antenna frequencies and the need for a combination of algorithms to improve the accuracy of the results.

\section{REFERENCES}

[1] A. Ghosh, R. Ratasuk, B. Mondal, N. Mangalvedhe, and T. Thomas, "LTE-advanced: Next-generation wireless broadband technology," IEEE Wirel. Commun., 2010.

[2] H. J. Visser and R. J. M. Vullers, "RF energy harvesting and transport for wireless sensor network applications: Principles and requirements," Proceedings of the IEEE. 2013.

[3] I. Mujahidin, S. H. Pramono, and A. Muslim, "5.5 Ghz Directional Antenna with 90 Degree Phase Difference Output," 2018.

[4] D. M. Pozar, "New results for minimum Q, maximum gain, and polarization properties of electrically small arbitrary antennas," in 3rd European Conference on Antennas and Propagation, 2009.

[5] R. Yuwono and I. Mujahidin, "Rectifier using UWB microstrip antenna as electromagnetic energy harvester for GSM, CCTV and Wi-Fi transmitter," J. Commun., 2019.

[6] D. J. Norris, Beginning Artificial Intelligence with the Raspberry Pi. 2017.

[7] I. Mujahidin, "Directional 1900 Mhz Square Patch Ring Slot Microstrip Antenna For WCDMA," JEEMECS (Journal Electr. Eng. Mechatron. Comput. Sci., 2019.

[8] Y. J. Cheng, W. Hong, K. Wu, and Y. Fan, "A hybrid guided-wave structure of half mode substrate integrated waveguide and conductorbacked slotline and its application in directional couplers," IEEE Microw. Wirel. Components Lett., 2011.

[9] R. Yuwono, I. Mujahidin, A. Mustofa, and Aisah, "Rectifier using UFO microstrip antenna as electromagnetic energy harvester," Adv. Sci. Lett., 2015.

[10] C. E. Balanis, "Antenna Theory: Analysis and Design, 3rd Edition Constantine A. Balanis," Book. 2005.

[11] I. Mujahidin and B. F. Hidayatulail, "2.4 GHz Square Ring Patch With Ring Slot Antenna For Self Injection Locked Radar," JEEMECS (Journal Electr. Eng. Mechatron. Comput. Sci., vol. 2, no. 2, 2019.

[12] A. Tavanaei, M. Ghodrati, S. R. Kheradpisheh, T. Masquelier, and A. Maida, "Deep learning in spiking neural networks," Neural Networks. 2019.

[13] D. A. Prasetya, A. Sanusi, G. Chandrarin, E. Roikhah, I. Mujahidin, and R. Arifuddin, "Small and Medium Enterprises Problem and Potential Solutions for Waste Management," J. Southwest Jiaotong Univ., vol. 54, no. 6, 2019.

[14] R. Shokri and V. Shmatikov, "Privacy-preserving deep learning," in 2015 53rd Annual Allerton Conference on Communication, Control, and Computing, Allerton 2015, 2016.

[15] D. A. Prasetya, A. Sanusi, G. Chandrarin, E. Roikhah, I. Mujahidin, and R. Arifuddin, "Community Culture Improvisation Regarding Waste Management Systems and Per Capita Income Increase," J. Southwest Jiaotong Univ., vol. 54, no. 6, 2019.

[16] C. Cortes, X. Gonzalvo, V. Kuznetsov, M. Mohri, and S. Yang, "AdaNet: Adaptive structural learning of artificial neural networks," in 34th International Conference on Machine Learning, ICML 2017, 2017.

[17] M. Kalash, M. Rochan, N. Mohammed, N. D. B. Bruce, Y. Wang, and F. Iqbal, "Malware Classification with Deep Convolutional Neural Networks," in 2018 9th IFIP International Conference on New Technologies, Mobility and Security, NTMS 2018 - Proceedings, 2018.

[18] Y. Bengio, "Learning deep architectures for AI," Found. Trends Mach. Learn., 2009.

[19] D. L. Deng, X. Li, and S. Das Sarma, "Quantum entanglement in neural network states," Physical Review X. 2017.

[20] M. M. Bronstein, J. Bruna, Y. Lecun, A. Szlam, and P. Vandergheynst, 
"Geometric Deep Learning: Going beyond Euclidean data," IEEE Signal Processing Magazine. 2017.

[21] Y. Guo, Y. Liu, A. Oerlemans, S. Lao, S. Wu, and M. S. Lew, "Deep learning for visual understanding: A review," Neurocomputing, 2016.

[22] A. Rajkomar et al., "Scalable and accurate deep learning with electronic health records," npj Digit. Med., 2018.
[23] I. Mujahidin, D. A. Prasetya, A. B. Setywan, and P. S. Arinda, "Circular Polarization 5.5 GHz Double Square Margin Antenna in the Metal Framed Smartphone for SIL Wireless Sensor," in 2019 International Seminar on Intelligent Technology and Its Applications (ISITIA), 2019, pp. 1-6.

APPENDIX

The appendix contains sample results from the results of experiments that have been carried out. The sample provides an overview of the accuracy of the prediction algorithm used. Thus algorithmic implementation can be implemented more efficiently in the various antenna sizes that are proposed. From some of the samples displayed, all value is the good agreements that based on existing minimum standard parameters.

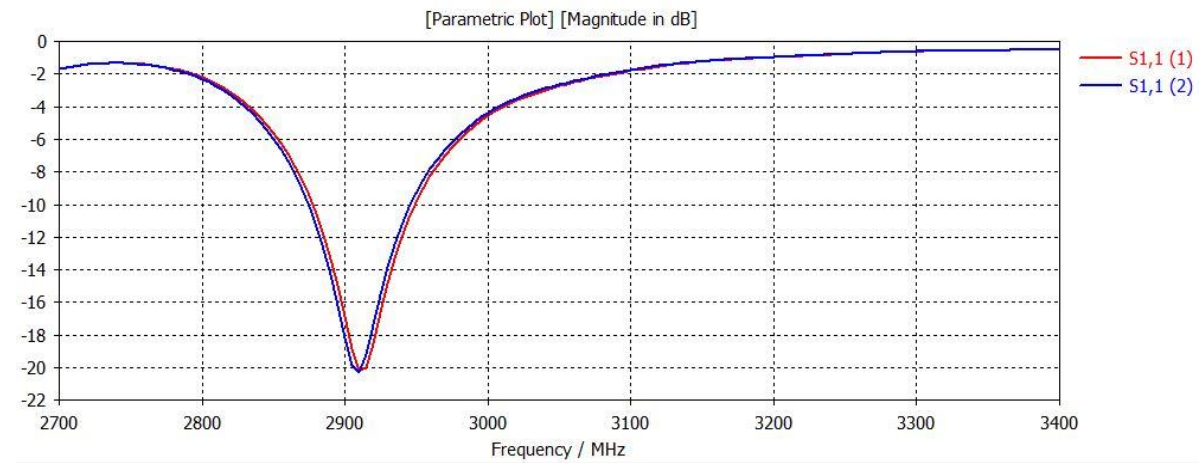

(a)

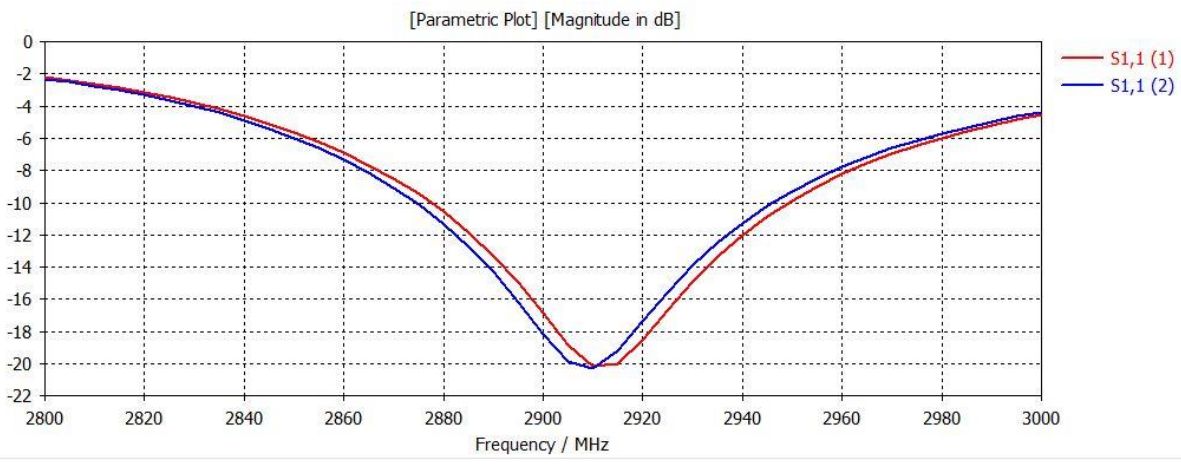

(b)

Fig. 4. Comparison of prediction and truth for $2913 \mathrm{MHz}$ input

TABle II. Prediction Result and Truth of the Testing Data

\begin{tabular}{|c|c|c|c|c|c|c|c|c|c|c|c|c|}
\hline \multirow[t]{2}{*}{ No } & \multicolumn{2}{|c|}{$\begin{array}{l}\text { Ground Plane } \\
\text { Height }\end{array}$} & \multicolumn{2}{|c|}{$\begin{array}{l}\text { Triangle Side } \\
\text { Length }\end{array}$} & \multicolumn{2}{|c|}{ Circle Diameters } & \multicolumn{2}{|c|}{ S-Parameter } & \multicolumn{2}{|l|}{ Gain } & \multicolumn{2}{|c|}{ Directivity } \\
\hline & $T$ & $\mathbf{P}$ & $\mathbf{T}$ & $\mathbf{P}$ & $T$ & $\mathbf{P}$ & $\mathbf{T}$ & $\mathbf{P}$ & $\mathbf{T}$ & $\mathbf{P}$ & $T$ & $\mathbf{P}$ \\
\hline 1 & 40 & 40 & 20 & 20 & 16 & 15.981 & -20.820 & -18.981 & 4.8 & 4.678 & 4.8 & 5.066 \\
\hline 2 & 40 & 40 & 20 & 20 & 16.1 & 16.077 & -20.510 & -19.120 & 4.8 & 4.688 & 4.84 & 5.085 \\
\hline 3 & 40 & 40 & 20 & 20 & 16.2 & 16.212 & -20.270 & -19.314 & 4.32 & 4.701 & 4.93 & 5.113 \\
\hline 4 & 40 & 40 & 20 & 20 & 16.3 & 16.270 & -20.100 & -19.397 & 4.47 & 4.706 & 4.98 & 5.125 \\
\hline 5 & 40 & 40 & 20 & 20 & 16.4 & 16.385 & -20.270 & -19.564 & 4.47 & 4.718 & 5.04 & 5.149 \\
\hline 6 & 40.01 & 40.01 & 20 & 20 & 17.3 & 17.333 & -20.230 & -20.076 & 4.4 & 4.821 & 5.42 & 5.364 \\
\hline 7 & 40.01 & 40.01 & 20 & 20 & 17.4 & 17.431 & -20 & -21.215 & 4.83 & 4.830 & 5.41 & 5.384 \\
\hline 8 & 40.01 & 40.009 & 20 & 20 & 17.5 & 17.556 & -22.130 & -21.395 & 4.87 & 4.843 & 5.46 & 5.410 \\
\hline 9 & 40.01 & 40.009 & 20 & 20 & 17.6 & 17.662 & -21.950 & -21.548 & 4.96 & 4.853 & 5.5 & 5.432 \\
\hline 10 & 40.01 & 40.009 & 20 & 20 & 17.7 & 17.710 & -21.580 & -21.617 & 4.96 & 4.858 & 5.5 & 5.442 \\
\hline 11 & 40.02 & 40.009 & 20 & 20 & 17.8 & 17.855 & -21.227 & -21.825 & 4.96 & 4.873 & 5.54 & 5.471 \\
\hline 10 & 40.02 & 40.009 & 20 & 20 & 17.9 & 17.942 & -21.450 & -21.950 & 4.92 & 4.880 & 5.62 & 5.489 \\
\hline 11 & 40.02 & 40.009 & 20 & 20 & 18 & 18.040 & -22.090 & -22.103 & 4.89 & 4.891 & 5.61 & 5.511 \\
\hline Sum & \multicolumn{2}{|l|}{0.306} & \multicolumn{2}{|l|}{0} & \multicolumn{2}{|c|}{1.254} & \multicolumn{2}{|l|}{4.52} & \multicolumn{2}{|l|}{0.69} & \multicolumn{2}{|c|}{0.391} \\
\hline MAE & \multicolumn{2}{|l|}{0.027} & \multicolumn{2}{|l|}{ 0 } & \multicolumn{2}{|c|}{0.114} & \multicolumn{2}{|l|}{0.410} & \multicolumn{2}{|l|}{0.062} & \multicolumn{2}{|c|}{0.035} \\
\hline
\end{tabular}

